\title{
Multi-frequency observations of Swift J1626.6-5156
}

\author{
P. Reig ${ }^{1,2}$, E. Nespoli ${ }^{3,4}$, J. Fabregat ${ }^{3}$, and R. E. Mennickent ${ }^{5}$
}

\author{
1 IESL, Foundation for Research \& Technology-Hellas 71110 Heraklion, Crete, Greece \\ e-mail: pau@physics.uoc.gr \\ 2 Department of Physics and Institute of Theoretical \& Computational Physics, University of Crete, 71003 Heraklion, Greece \\ 3 Observatorio Astronómico Universidad de Valencia, C/ Catedrático Agustín Escardino Benlloch 7, 46980 Paterna, Valencia, Spain \\ e-mail: [elisa; juan. fabregat]@uv.es \\ 4 Valencian International University, Pr. C/ José Pradas Gallen, 12006 Castellón de la Plana, Spain \\ 5 Departamento de Astronomía, Universidad de Concepción, Chile, Casilla 160-C, Concepción, Chile \\ e-mail: rmennick@udec.cl
}

Received 20 May 2011 / Accepted 14 July 2011

\begin{abstract}
Context. Swift J1626.6-5156 is an X-ray pulsar that was discovered in December 2005 during an X-ray outburst. Although the X-ray data suggest that the system is a high-mass X-ray binary, very little information exists on the nature of the optical counterpart.

Aims. We investigate the emission properties of the optical counterpart in the optical and near-IR bands and the long-term X-ray variability of the system to determine unambiguously the nature of this X-ray pulsar.

Methods. We have performed an X/optical/IR analysis of Swift J1626.6-5156. We have analysed all RXTE observations since its discovery, archived optical spectroscopic and photometric data and obtained near-IR spectra for the first time. X-ray energy spectra were fitted with models composed of a combination of photoelectric absorption, a power law with high-energy exponential cutoff and a Gaussian line profile at $6.5 \mathrm{keV}$ and an absorption edge at around $9 \mathrm{keV}$. X-ray power spectra were fitted with Lorentzian profiles. We identified and measured the equivalent width and relative intensity of the spectral features in the optical and infrared spectra to determine the spectral type of the optical counterpart.

Results. The K-band spectrum shows He I $\lambda 20581 \AA$ and H I $\lambda 21660 \AA$ (Brackett-gamma) in emission, which confines the spectral type of the companion to be earlier than B2.5. The $H$-band spectrum exhibits the $\mathrm{HI} \mathrm{Br}-18-11$ recombination series in emission. The most prominent feature of the optical band spectrum is the strong emission of the Balmer line H $\alpha$. The 4000-5000 A spectrum contains He II and numerous He I lines in absorption, indicating an early B-type star. The source shows three consecutive stages characterised by different types of variability in the X-ray band: a smooth decay after the peak of a major outburst, high-amplitude flaring variability (reminiscent of type I oytbursts) and quiescence. We observed that the spectrum becomes softer as the flux decreases and that this is a common characteristic of the X-ray emission for all observing epochs. An emission line feature at $\sim 6.5 \mathrm{keV}$ is also always present.

Conclusions. The X-ray/optical/IR continuum and spectral features are typical of an accreting X-ray pulsar with an early-type donor. The long-term X-ray variability with its major and minor outbursts and quiescent emission is also characteristic of hard X-ray transients. We conclude that Swift J1626.6-5156 is a Be/X-ray binary with a B0Ve companion located at a distance of $\sim 10 \mathrm{kpc}$.
\end{abstract}

Key words. binaries: close - stars: neutron - X-rays: binaries - stars: emission-line, Be

\section{Introduction}

Swift J1626.6-5156 is a hard X-ray transient whose actual nature is uncertain. It was discovered on 18 December 2005 by Swift/BAT when its X-ray emission was showing short-term flaring episodes (Palmer et al. 2005). Immediate after the first detection by Swift, the source was observed by RXTE, which confirmed the presence of an X-ray pulsar with $P_{\text {spin }}=15.377$ $\mathrm{s}$ and strong variations of the pulse fraction during the flares (Markwardt \& Swank 2005; Belloni et al. 2006). In a subsequent observation with Swift/XRT, the X-ray position was refined to RA (J2000): 16:26:36.24, Dec (J2000): -51:56:33.5, with a 90\% error radius of 3.5" (Campana et al. 2006). The optical and infrared observations of the only counterpart so far proposed are contradictory. While optical spectroscopy suggests a Be star (Negueruela \& Marco 2006), infrared observations seem to indicate a late-type object (Rea et al. 2006). The $\gamma$-ray mission INTEGRAL also detected Swift J1626.6-5156 about a year after its discovery, but no X-ray flares were observed (Tarana et al. 2006).

The first detailed study of Swift J1626.6-5156 was carried out by Reig et al. (2008), who found that the duration of the flares was a few hundred seconds and that the X-ray intensity increased by a factor of 3.5 during the flares. The authors also presented evidence (although not conclusive) in favour of a highmass X-ray binary classification of the system. The determination of the nature of the system is very important because the flares seen in Swift J1626.6-5156 would constitute the shortest events of this kind ever reported in a high-mass X-ray binary.

In a recent paper, Baykal et al. (2010, see also Icdem et al. 2011) performed a pulse frequency analysis and reported the discovery of the orbital parameters of the system. The authors also studied the evolution of the neutron star spin period throughout the outburst. The periodic trend of pulse frequencies yielded an orbital period of $P_{\text {orb }}=132.89 \pm 0.03$ days and an eccentricity of $e=0.08 \pm 0.01$. Pulse-phase spectroscopy has also been addressed by Reig et al. (2008), while a preliminary analysis 


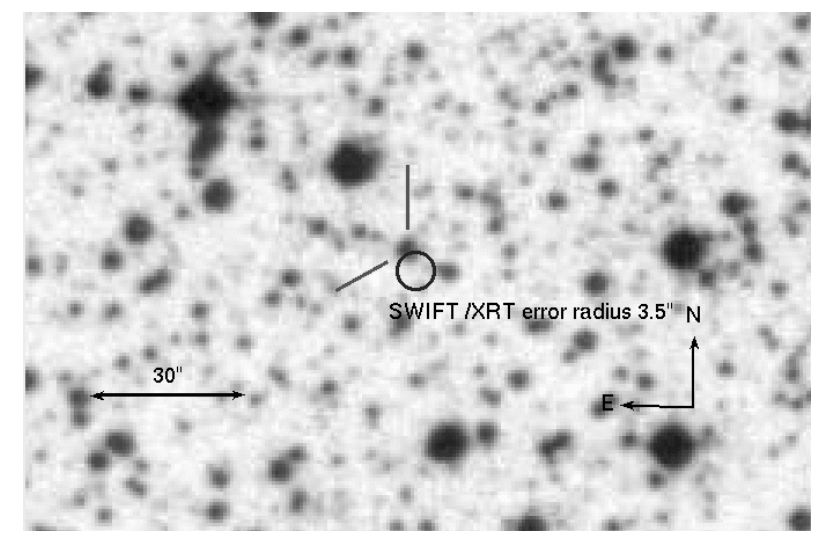

Fig. 1. Finding chart with the identification of the optical counterpart to Swift J1626.6-5156. The image was downloaded from the Aladin Sky Atlas.

Table 1. Optical/infrared apparent magnitudes of the optical counterpart to Swift J1626.6-5156 from the NOMAD catalogue.

\begin{tabular}{ccccccc}
\hline \hline$B$ & $V$ & $R$ & $I$ & $J$ & $H$ & $K$ \\
\hline 16.81 & 15.54 & 15.81 & 14.27 & 13.44 & 12.95 & 12.54 \\
\hline
\end{tabular}

Notes. Note that $B, V, R$, and $I$ are photographic magnitudes.

Table 2. $H$ - and $K$-band line identifications and measured equivalent widths for 2MASS J16263652-5156305.

\begin{tabular}{lcc}
\hline \hline $\begin{array}{l}\text { Spectral } \\
\text { feature }\end{array}$ & $\begin{array}{c}\text { Wavelength } \\
(\mu \mathrm{m})\end{array}$ & $\begin{array}{c}\text { Equivalent } \\
\text { width }(\AA)\end{array}$ \\
\hline $\mathrm{Br}-10$ & 1.737 & $-7.8 \pm 0.3$ \\
$\mathrm{Br}-11$ & 1.678 & $-7.9 \pm 0.5$ \\
$\mathrm{Br}-12$ & 1.641 & $-8.3 \pm 0.5$ \\
$\mathrm{Br}-13$ & 1.611 & $-7.0 \pm 0.5$ \\
$\mathrm{Br}-14$ & 1.588 & $-9.7 \pm 0.6$ \\
$\mathrm{Br}-15$ & 1.570 & $-8.3 \pm 0.5$ \\
$\mathrm{Br}-16$ & 1.556 & $-7.8 \pm 0.2$ \\
$\mathrm{Br}-17$ & 1.544 & $-8.0 \pm 0.3$ \\
$\mathrm{Br}-18$ & 1.535 & $-6.2 \pm 0.3$ \\
$\mathrm{He} \mathrm{I}$ & 2.058 & $-9.9 \pm 0.4$ \\
$\mathrm{Br} \gamma$ & 2.166 & $-8.8 \pm 0.3$ \\
\hline
\end{tabular}

of the evolution of the pulse profiles can be found in Baykal et al. (2011). Here we present a detailed X-ray spectral and timing analysis of the entire 2006 outburst and subsequent flaring emission. The X-ray varibility that followed the major outburst is reminiscent of the so-called type-I outburst, seen in many Be/X-ray binaries (see e.g., Wilson et al. 2002). However, unlike typical Be/X-ray binaries, the frequency of the outbursts observed in Swift J1626.6-5156 does not always equal the orbital period (Baykal et al. 2010). For this reason, we shall refer to these quasiperiodic enhancements of the X-ray intensity as flares (not to be confused with the three short-term flares reported by Reig et al. 2008). We also analysed data during the X-ray quiescent state that followed the flaring state and present new optical and infrared observations. Our aim is to solve the nature of this unusual accreting X-ray pulsar.
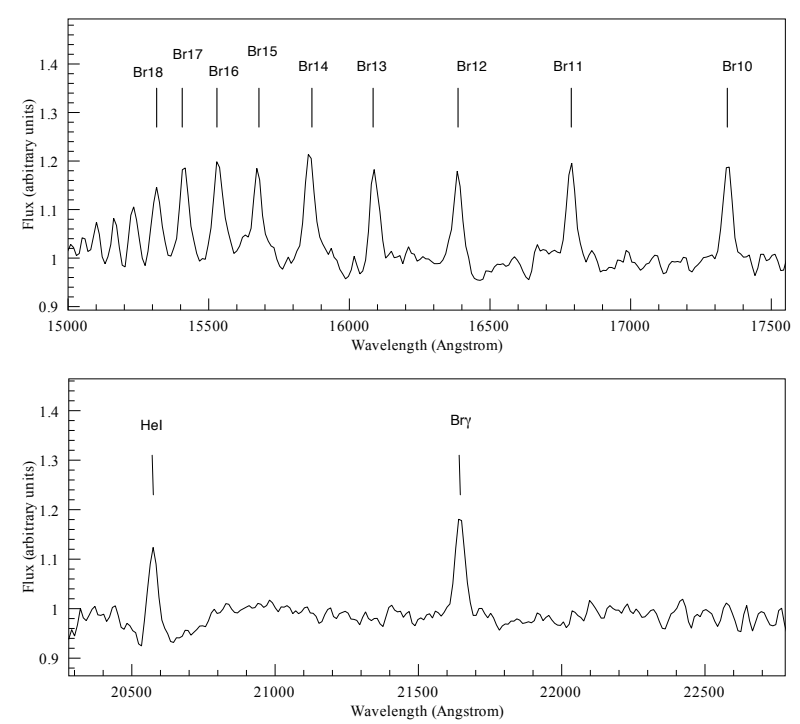

Fig. 2. $H$ - and $K$-band spectra of the infrared counterpart to Swift J1626.6-5156. Helium and hydrogen lines are clearly seen in emission.

\section{Optical and infrared observations}

\subsection{Near-IR observations}

Figure 1 shows a digital image of the field around the best X-ray position with the identification of the optical counterpart. NearIR data were obtained in visiting mode in July 2010 at the European Southern Observatory (ESO). The employed instrument was the SofI spectrograph (Moorwood et al. 1998), on the $3.5 \mathrm{~m}$ New Technology Telescope (NTT) at La Silla, Chile. We used the long-slit spectroscopy mode at low resolution $(R=588)$ with the $1.53-2.52 \mu \mathrm{m}$ grism and $1^{\prime \prime}$ width slit. The instrument large-field objective provided a FOV of $4.92^{\prime} \times 4.92^{\prime}$. The sky had thin cirri and seeing averaged between $0.9^{\prime \prime}$ and $1.3^{\prime \prime}$.

With the aim of ensuring accurate removal of atmospheric features from the spectrum, we followed a strategy similar to that outlined by Clark \& Steele (2000). At the telescope, we observed an A0IV standard star immediately before the target and a G3 V immediately after it to obtain very small differences in airmass (differences between 0.02 and 0.04 airmasses were accomplished). To compute the telluric features in the region of the H I $21661 \AA$ (Brackett- $\gamma$ line, or Br $\gamma$ ), which is the only nontelluric feature in the A-star spectra, we employed the observed G-star spectra divided by the solar spectrum ${ }^{1}$ properly degraded in resolution. The dispersion solution obtained for the SofI spectra was also applied. Then the spectra of the A-star, G-star and the solar one were aligned in wavelength space. For the $K$ spectral region, a telluric spectrum was obtained by patching the ratio between the G-star and the solar spectrum in the $\mathrm{Br} \gamma$ region into the A-star spectrum (we selected the range 21 590-21739 $\AA$ ). For the $H$ spectral region, the ratio between the G-star and the solar spectrum was employed as the telluric spectrum.

Data reduction was performed using the IRAF ${ }^{2}$ package, following the standard procedure. We first corrected for the interquadrant row cross-talk, a feature that affects the SofI detector;

\footnotetext{
1 We used the NSO/Kitt Peak FTS solar spectrum, produced by NSF/NOAO

${ }^{2}$ IRAF is distributed by the National Optical Astronomy Observatories which is operated by the Association of Universities for Research in Astronomy, Inc. under contract with the National Science Foundation.
} 
Table 3. Log of the optical spectroscopic observations.

\begin{tabular}{lcllccc}
\hline \hline Date & MJD & $\begin{array}{l}\text { Instrument } \\
\text { set-up }\end{array}$ & $\begin{array}{l}\text { Grism } \\
\text { number }\end{array}$ & $\begin{array}{c}\text { Wavelength } \\
\text { range }(\AA)\end{array}$ & $\begin{array}{c}E W(\mathrm{H} \alpha) \\
(\AA)\end{array}$ & $\begin{array}{c}E W(\mathrm{H} \beta) \\
(\AA)\end{array}$ \\
\hline $2006-02-15$ & 53782.359 & EMMI/NTT & grism\#6 & $5700-8700$ & $-40 \pm 2$ & - \\
$2007-09-17$ & 54360.984 & EFOSC/3P6 & grism\#14 & $3095-5085$ & - & $-4.3 \pm 0.5$ \\
$2007-09-17$ & 54361.008 & EFOSC/3P6 & grism\#18 & $4700-6770$ & $-45 \pm 2$ & $-5.0 \pm 0.9$ \\
$2007-09-18$ & 54361.993 & EFOSC/3P6 & grism\#3 & $3050-6100$ & - & $-4.5 \pm 0.5^{b}$ \\
$2007-09-18$ & 54362.025 & EFOSC/3P6 & grism\#14 & $3095-5085$ & - & $-4.2 \pm 0.5^{a}$ \\
$2009-06-01$ & 54983.078 & EFOSC/NTT & grism\#13 & $3685-9315$ & $-45 \pm 2$ & $-5.1 \pm 0.9$ \\
\hline
\end{tabular}

Notes. ${ }^{a}$ Average of three measurements; ${ }^{b}$ Average of two measurements.

we then applied sky subtraction; we employed dome flat-fields and extracted the one-dimensional spectrum. Wavelength calibration was accomplished using xenon and neon lamp spectra. Spurious features such as cosmic rays or bad pixels were removed by interpolation, when necessary. The reduced spectra, one covering the $H$-band, one the $K$-band, were normalized by dividing them by a fitted polynomial continuum. We finally corrected for telluric absorption, dividing each scientific spectrum by its corresponding telluric spectrum, obtained as described above. A scale and a shift factor were applied to the telluric spectrum to best correct for the airmass difference and the possible wavelength shift; the optimum values for these parameters were obtained using an iterative procedure that minimizes the residual noise. The final $H$ - and $K$-band spectra are shown in Fig. 2.

\subsection{Optical spectra}

Optical spectroscopic data were retrieved from the ESO archive facility. Table 3 shows the log of the optical spectroscopic observations, summarising the instrumental set-up and giving the equivalent width of the $\mathrm{H} \alpha$ and $\mathrm{H} \beta$ lines. The EMMI observation corresponds to that reported by Negueruela \& Marco (2006), while the EFOSC observations are unpublished: programme IDs 079.D-0371(A) and 083.D-0110(A). Data reduction was performed using the IRAF packages, following the standard procedure.

\section{X-ray observations and data analysis}

We analysed data obtained by all three instruments onboard RXTE (Bradt et al. 1993): the All-Sky Monitor (ASM) data consist of daily flux averages in the energy range $1.3-12.1 \mathrm{keV}$. The Proportional Counter Array (PCA) covers the lower part of the energy range $2-60 \mathrm{keV}$, and consists of five identical coaligned gas-filled proportional units giving a total collecting area of $6500 \mathrm{~cm}^{-2}$ and provides an energy resolution of $18 \%$ at $6 \mathrm{keV}$. The High Energy Timing Experiment (HEXTE) consists of two clusters of four NaI/CsI scintillation counters, with a total collecting area of $2 \times 800 \mathrm{~cm}^{2}$, sensitive in the $15-250 \mathrm{keV}$ band with a nominal energy resolution of $15 \%$ at $60 \mathrm{keV}$. Data taken during satellite slews, passage through the South Atlantic Anomaly and Earth occultation were removed. The overall analysed on-source time amounts to $496.3 \mathrm{ks}$.

The mission-specific packages of Heasarc FTOOLS (version 6.6.3) were employed to perform data reduction, while XSPEC v12. $6^{3}$ was used for spectral analysis. For each observation we obtained an average energy spectrum and a power spectrum.

\footnotetext{
$\overline{{ }^{3} \text { http://heasarc.gsfc.nasa.gov/docs/xanadu/xspec/ }}$
}

The energy spectra were generated from standard mode data, namely, the PCA Standard 2 of PCU2 and HEXTE "FS58" cluster B data, which have a time resolution of $16 \mathrm{~s}$ and cover the 2-60 keV range with 129 channels and the $15-250 \mathrm{keV}$ with 64 channels, respectively. All spectra were backgroundsubtracted. A systematic error of $0.6 \%$ was added in quadrature to the PCA spectra to account for systematic errors. The power spectra were generated by computing Fourier transforms of 128-s segments of the $2^{-6}$-s binned light curves in the energy range 2-15 keV (PCA channels 0-35) and averaging them together. The final power spectra were logarithmically rebinned in frequency and corrected for dead-time effects according to the prescription given in Nowak et al. (1999). Power spectra were normalized such that the integral over a given frequency range equals to the squared fractional rms amplitude (Belloni \& Hasinger 1990; Miyamoto et al. 1991).

To fit the energy spectra, we used a model composed by a combination of photoelectric absorption, a power law with highenergy exponential cutoff, a Gaussian line profile at $6.5 \mathrm{keV}$ and an absorption edge at around $9 \mathrm{keV}$ to account for $\mathrm{Fe} \mathrm{K}$ fluorescence. We did not find evidence for a cyclotron absorption feature, commonly seen in other accreting X-ray pulsars. The power spectra were fitted using Lorentzian profiles only. The main peak of the X-ray pulsations and two to three of its harmonics are clearly seen in the power spectra. These peaks were fitted with narrow Lorentzians (the width was normally fixed at $\sim 0.001 \mathrm{~Hz}$ ). The broad-band noise was fitted with two zerocentred Lorentzians. The brighter observations required one extra Lorentzian to account for the noise above $\sim 7 \mathrm{~Hz}$.

\section{The optical counterpart}

\subsection{Spectral type}

The information on the optical counterpart to Swift J1626.65156 is very scarce. To the authors' knowledge no optical photometric observations are published. The only dedicated observations are those reported in the Astronomer's Telegrams ATel\#713 (Rea et al. 2006), who obtained infrared photometric magnitudes and ATel\#739 (Negueruela \& Marco 2006), who performed spectroscopic observations and reported the strong presence of $\mathrm{H} \alpha$ in emission.

Information on photometric magnitudes and colours can be found in astronomical catalogues. We searched for possible optical/infrared counterparts in the $\mathrm{NOMAD}^{4}$ catalogue around the best-fit X-ray position (Campana et al. 2006). The

\footnotetext{
${ }^{4}$ The NOMAD catalogue is a facility provided by the US Naval Observatory and contains astrometric and photometric data for about 1.1 billion stars derived from the Hipparcos, Tycho-2, UCAC2, YellowBlue 6, and USNO-B catalogs for astrometry and optical photometry,
} 

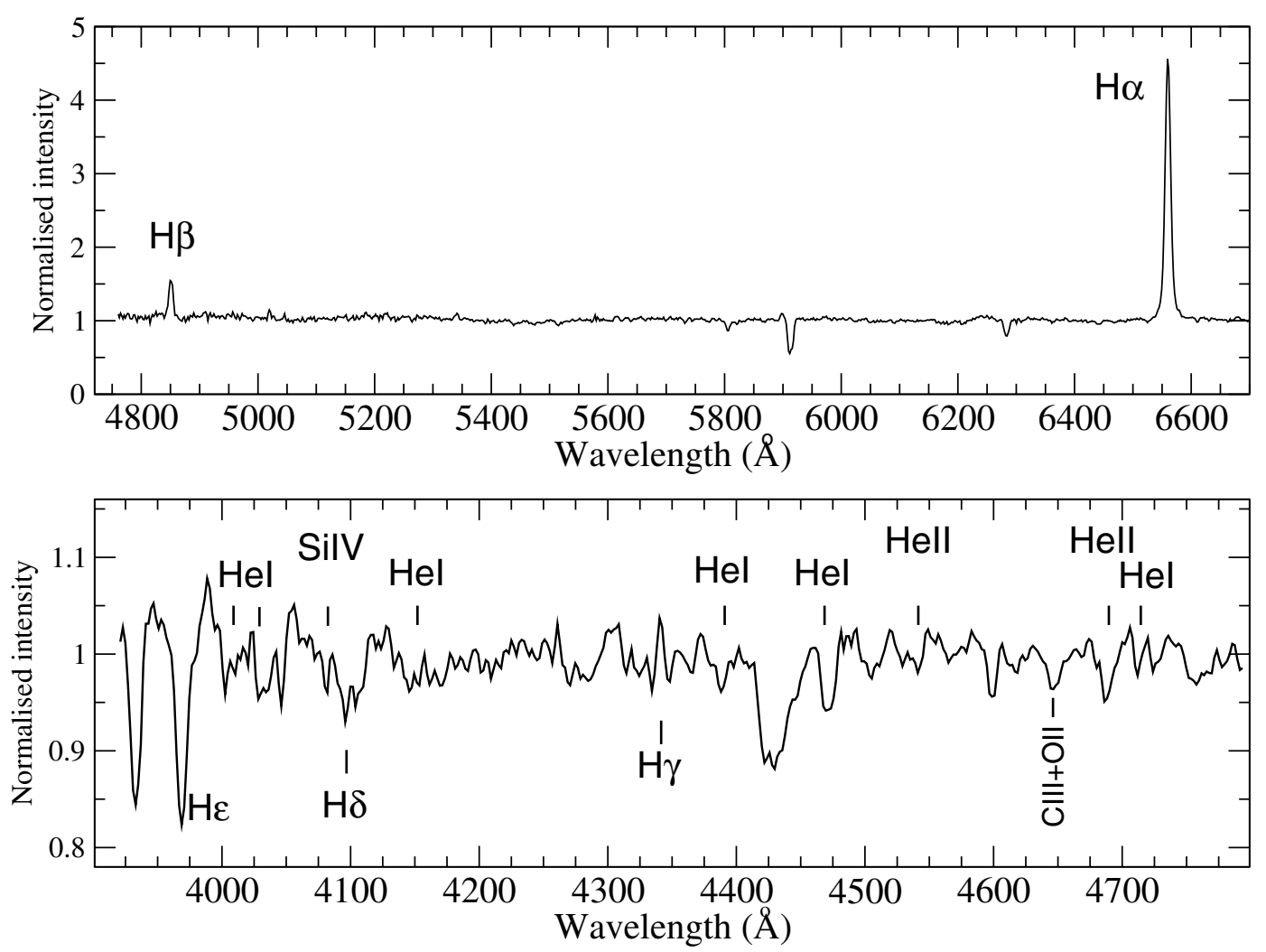

Fig. 3. Optical spectrum in the classification region. The identified lines are He I $\lambda 4009, \lambda 4026, \lambda 4144, \lambda 4387, \lambda 4471, \lambda 4713 \AA$; He II $\lambda 4541$ and $\lambda 4686 \AA$; Si IV $\lambda 4089 \AA$ A the C III+O II blend $\lambda 4650 \AA$.

optical counterpart to Swift J1626.6-5156 is the star named as NOMAD1-0380-0705569 (=2MASS16263652-5156305 = USNO-B1.0 0380-0649488) and it is shown in Fig. 1. The selected star is the only one exhibiting significant infrared (Rea et al. 2006) and $\mathrm{H} \alpha$ (Negueruela \& Marco 2006) emission within the Swift/XRT error circle or its vicinity, which are the signatures of the vast majority of optical counterparts in accreting $\mathrm{X}$-ray pulsars. Table 1 gives the catalogued photometric magnitudes.

The low-resolution $K$-band spectrum is almost featureless, except for the presence of He I $\lambda 20581 \AA$, slightly affected by a residual telluric absorption and H I $\lambda 21660 \AA$ (Brackett- $\gamma$, or $\mathrm{Br} \gamma$ ), which appear strongly in emission. These two lines are typical of Be-stars and confine the spectral type of the companion star to be earlier than B2.5 (Clark \& Steele 2000). The $H$-band spectrum is more complex, with the $\mathrm{HI} \mathrm{Br-18-11} \mathrm{re-}$ combination series in emission. All observed features of the $K$ band spectrum are typical of Be stars, while they are absent, or observed in absorption in normal (non-emission) OB stars. The observed features with measured equivalent widths are shown in Table 2.

By comparison with spectral atlases (Clark \& Steele 2000; Steele \& Clark 2001), the optical counterpart to Swift J1626.65156 can be classified as a B0-2Ve star, confirming the nature of the system as a high-mass X-ray binary. Note that all spectroscopically identified optical companions of $\mathrm{Be} / \mathrm{X}$-ray binaries in the Milky Way have spectral types in the narrow range O9-B2, with a peak around B0 (see e.g., Fig. 6 in Antoniou et al. 2009).

supplemented by 2MASS near-infrared photometry. The primary aim of NOMAD is to help users retrieve the best currently available astrometric data for any star in the sky by providing these data in one place.
Based on JHK photometry, Rea et al. (2006) suggested that the optical counterpart to Swift J1626.6-5156 could not be an early-type star because after de-reddening using the X-ray hydrogen column density $\left(N_{\mathrm{H}}=0.9 \times 10^{21} \mathrm{~cm}^{-2}\right)$, the infrared magnitudes and colours were too faint to be consistent with an OB star. The value of $N_{\mathrm{H}}$ corresponded to a Swift/XRT observations reported by Campana et al. (2006). However, the actual value of $N_{\mathrm{H}}$ reported in Campana et al. (2006) is an order of magnitude higher than that used by Rea et al. (2006). We repeated the computation of the intrinsic colour of the source, employing the Swift/XRT $N_{\mathrm{H}}$ value from Campana et al. (2006), $N_{\mathrm{H}}=(9.4 \pm 1.0) \times 10^{21} \mathrm{~cm}^{-2}$, which is more reliable than our RXTE measurement because of the softer energy range sensitivity. This implies a visual interstellar extinction $A_{\mathrm{V}}=4.25 \pm 0.45 \mathrm{mag}$ (Güver \& Özel 2009), which converts into an interstellar IR colour excess $E^{\text {is }}(H-K)=0.063 \times$ $A_{\mathrm{V}}=0.27 \pm 0.03 \mathrm{mag}$ (Rieke \& Lebofsky 1985). Note that the dense circumstellar material surrounding the Be star introduces extra reddening that cannot be ignored, especially at long wavelengths (Dachs et al. 1988; Dougherty et al. 1994). Hence $E^{\text {tot }}(H-K)=E^{\text {is }}(H-K)+E^{\mathrm{cs}}(H-K)$. The reddening caused by the disc can be estimated from the relationship between infrared excess and the $\mathrm{H} \alpha$ equivalent width from Howells et al. (2001), who derived $E^{\mathrm{cs}}(H-K)=0.006 E W(H \alpha)-0.030 \mathrm{mag}$. We do not know the state of the disc at the time that the infrared observations were measured, but if we assume that the $\mathrm{H} \alpha$ equivalent width was at the level reported here $\sim-40 \AA$, then $E^{\mathrm{cs}}(H-K)=0.21 \pm 0.02 \mathrm{mag}$ and the resulting intrinsic colour is $(H-K)_{0}=(H-K)_{2 \mathrm{MASS}}-E^{\mathrm{tot}}(H-K)=-(0.07 \pm 0.04) \mathrm{mag}$, consistent with an early-type star (Koornneef 1983). The errors were obtained by propagating the errors of the measurements. 


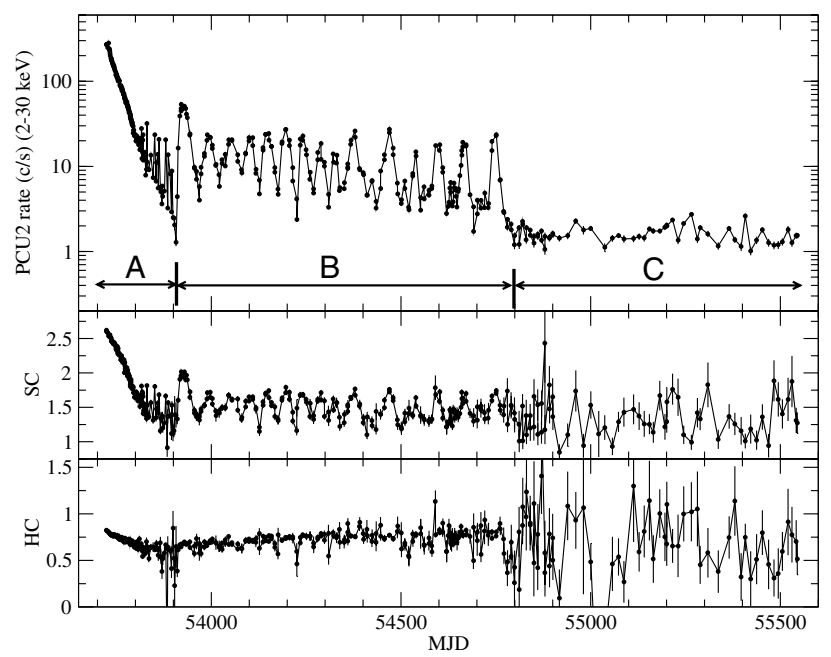

Fig. 4. X-ray light curve of Swift J1626.6-5156 showing five years worth of data. Each data point corresponds to the average count rate of the individual pointings. Also shown are the soft colour, $\mathrm{SC}=4$ $7 \mathrm{keV} / 2-4 \mathrm{keV}$ and hard colour, $\mathrm{HC}=10-15 \mathrm{keV} / 7-10 \mathrm{keV}$.

The September 2007 blue-end spectra allow us to refine the spectral type estimated from the near-IR data. Figure 3 shows the optical spectrum of Swift J1626.6-5156 in the traditional classification region (3800-4800 $\AA$ ). Note that emission affects the lines of the Balmer series: $\mathrm{H} \beta$ and $\mathrm{H} \gamma$ are in emission, while $\mathrm{H} \delta$ is partially filled-in with emission. The spectrum contains many He I lines $(\lambda 4009, \lambda 4026, \lambda 4144, \lambda 4387, \lambda 4471, \lambda 4713 \AA)$, indicating an early B-type star. The presence of He II ( $\lambda 4541$ and $\lambda 4686 \AA$ ) indicates that the spectral type of the optical star is earlier than B1. Specifically, He II $\lambda 4686 \AA$ is last seen at B0.5-B0.7 (Walborn \& Fitzpatrick 1990). On the other hand, the weakness of He II $\lambda 4541 \AA$ relative to He I $\lambda 4471 \AA$ indicates a spectral type later than $\mathrm{O} 9$.

With regard to the luminosity classification, the weakness of Si III $\lambda 4552-68 \AA$ and the greater intensity of He II $\lambda 4686 \AA$ with respect to that of He I $\lambda 4713 \AA$ indicate a mainsequence star. Other luminosity indicators are the ratios of $\mathrm{He}$ lines to nearby metallic lines. Swift J1626.6-5156 shows He II $\lambda 4686 /$ CIII $\lambda 4650 \gtrsim 1$ and He I $\lambda 4144 / \mathrm{SiIV} 4089 \sim 1$. An evolved star would have ratios $<1$ and $\lesssim 1$, respectively.

A visual comparison of the Swift J1626.6-5156 spectrum (Fig. 3) with those of MK standards in the atlas by (Walborn \& Fitzpatrick 1990) reveals that the spectrum resembles that of the standard star $v$ Ori, a B0V star. Therefore we conclude that the optical counterpart to the X-ray accreting pulsar Swift J1626.65156 is a B0Ve star.

\subsection{Distance}

Although the uncertainty of the spectral type may introduce some extra uncertainty in the distance estimation, the main source of error in estimating the distance stems from the scattering of the intrinsic colours and absolute magnitudes associated to each spectral and luminosity class of the calibrations. Even for the same spectral type, variations of up to $1.5 \mathrm{mag}$ in the absolute magnitude (Wegner 2006) and of 0.04 mag in the intrinsic $(B-V)_{0}$ colours are found in studies from various authors (Johnson 1966; Wegner 1994). Jaschek \& Gómez (1998) analysed the absolute magnitude of about $100 \mathrm{MK}$ standards and

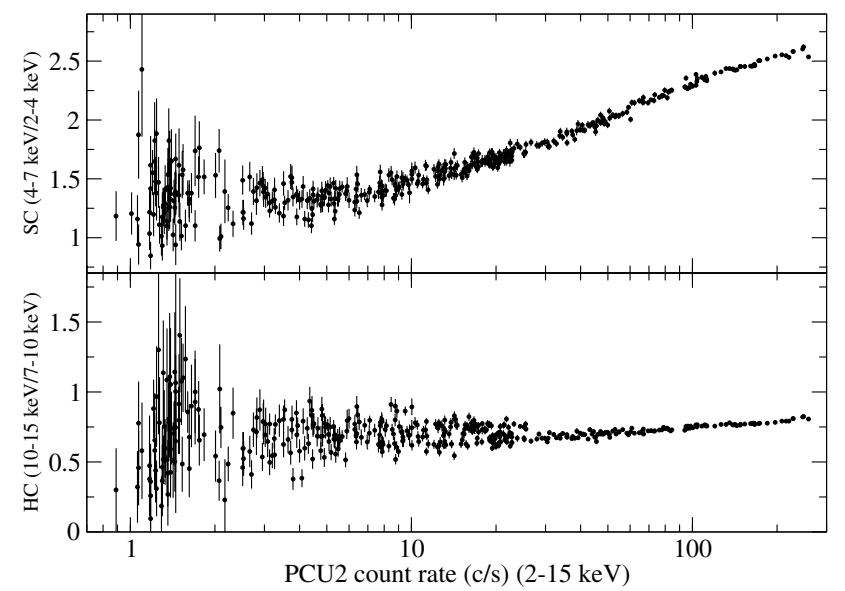

Fig. 5. Hardness-intensity diagram.

concluded that the intrinsic dispersion of the mean absolute magnitude amounts to $0.7 \mathrm{mag}$. We have adopted this value as the error on the absolute magnitude.

The BVRI magnitudes listed in Table 1 are photographic magnitudes extracted from the USNO-B1 and the YB6 surveys, and hence they are not directly comparable with the intrinsic colours and absolute magnitude calibrations of the Johnson-Cousins photometric system. This prevents us from using these magnitudes to compute the interstellar reddening and the distance to our target. We will approach this task by using the 2MASS JHK magnitudes also listed in Table 1.

Assuming that the optical counterpart to Swift J1626.6-5156 is a B0V star, then $(J-K)_{0}=-0.17$ mag (Koornneef 1983). The total IR excess is $E^{\text {tot }}(J-K)=1.07$. Part of this excess is caused by the circumstellar continuum emission of the Be star. We have calculated the circumstellar contribution to the IR excess to Be $E^{\mathrm{cs}}(J-K)=0.25 \mathrm{mag}$ from the equivalent width of the $\mathrm{H} \alpha$ line and the $\mathrm{H}$ - and $\mathrm{K}$-band spectral features by means of the formulae presented by Howells et al. (2001). Thus we obtained the interstellar IR excess as $E^{\text {is }}(J-K)=E^{\text {tot }}(J-K)-E^{\text {cs }}(J-K)=$ $0.82 \mathrm{mag}$. This implies a visual extinction of $A_{V}=4.7 \mathrm{mag}$ (Koornneef 1983), little higher, but consistent with the value obtained from the X-ray observations. The absolute magnitude of a B0V star is $M_{V}=-3.95$ mag (Wegner 2006), which can be converted into $M_{K}$ because $(V-K)_{0}=-0.85$ mag (Koornneef 1983 ); the intrinsic magnitude $K_{0}$ was computed as the difference of the 2MASS magnitude and the K-band extinction $A_{K}$ resulting from the relation $A_{\lambda} / E(J-K)=2.4(\lambda)^{-1.75}$, for $\lambda=2.2 \mu \mathrm{m}$ (Draine 1989). We finally calculated the distance from $M_{K}=K_{0}+5-5 \log d$, obtaining $d=10.7 \pm 3.5 \mathrm{kpc}$. This value must be considered as a lower limit, since we neglected the circumstellar contribution to $M_{K}$. The error was estimated assuming an error in the observed infrared magnitudes (hence $E(J-K))$ of $0.03 \mathrm{mag}$ and $0.7 \mathrm{mag}$ in the absolute magnitude.

\section{Long-term X-ray variability}

The long-term X-ray light curve of Swift J1626.6-5156, covering five years worth of data, from its discovery on 19 December 2005 (MJD 53723) until 14 December 2010 (MJD 55544) is shown in Fig. 4. This figure also shows the evolution of the X-ray colours defined by the ratio of the count rates in the energy range 4-7 keV over 2-4 keV (soft colour, SC) and 10-15 keV over 7-10 keV (hard colour, HC). The X-ray flux of Swift J1626.65156 decreased exponentially during the first 90 days of the 

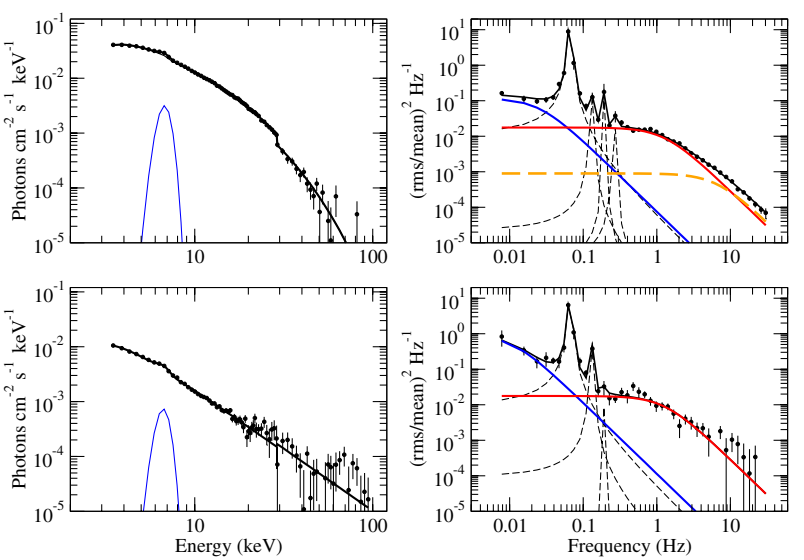

Fig. 6. Energy and power spectra at high (upper panels) and low (lower panels) X-ray flux. The X-ray flux is $\sim 4 \times 10^{-9}$ and $\sim 5 \times$ $10^{-10} \mathrm{erg} \mathrm{cm}^{-2} \mathrm{~s}^{-1}$, respectively.

observations. On 20 March 2006 (MJD 53814) the source began to exhibit low-amplitude X-ray flares, whose duration varied between 3-8 days. Nevertheless, the overall decrease in flux continued until 26 June 2006 (MJD 53912), where a large flare took place. After the large flare, the mean intensity increased to $\sim 10 \mathrm{c} \mathrm{s}^{-1} \mathrm{PCU}^{-1}$ and the X-ray flaring behaviour became more apparent. A total of 15 flares can be seen in Fig. 4. The time difference between the peak of the flares varied from $\sim 45$ days of the first half to $\sim 95$ days of the second half (Reig et al. 2008; Baykal et al. 2010).

On 29 November 2008 (MJD 54799) the X-ray count rate suddenly decreased by almost an order of magnitude to $\sim 1.5 \mathrm{c} \mathrm{s}^{-1} \mathrm{PCU}^{-1}$ and the large-amplitude flaring pattern ceased. The source entered a quiescent phase. Note that the source was observed daily until MJD 53827, every 2-4 days until MJD 53936, every 9-10 days until MJD 54900 and in a more irregular way every 15-20 days (but also including a few closer observations) until the end of the observations on MJD 55544.

The soft colour closely follows the evolution of the X-ray flux (Fig. 5). As the count rate increases, so does the SC. The $\mathrm{HC}$ also shows a positive correlation with flux. However, this is weaker than that at lower energies and it is evident only at higher count rates $\gtrsim 20 \mathrm{c} \mathrm{s}^{-1} \mathrm{PCU}^{-1}$. The correlation seems to extend to the lower count rate data but the scattering of the data points owing to poor statistics is too large below $\sim 3 \mathrm{c} \mathrm{s}^{-1} \mathrm{PCU}^{-1}$.

Given the richness in X-ray variability exhibited by Swift J1626.6-5156, we have defined three different time intervals and performed a separated analysis on each one. These periods correspond to the smooth decay part of the outburst, including the small-scale flaring period (MJD 53723-53912), the large-scale flaring (MJD 53912-54799) and the quiescent interval (MJD 54799-55544).

\subsection{Outburst decay (MJD 53723-53912)}

Swift J1626.6-5156 was discovered on 18 December 2005 when the source was near the peak of a major outburst. The X-ray flux of the first observations was $4.5 \times 10^{-9} \mathrm{erg} \mathrm{cm}^{-2} \mathrm{~s}^{-1}$ and decreased for the next $\sim 190$ days (interval A in Fig. 4). Figure 6 shows two representative energy and power spectra of the outburst decay at fluxes differing by about one order of magnitude. The spectral parameters changed smoothly as the outburst decayed (Fig. 7). However, at flux below $F_{\text {break }} \sim 1.5 \times$ $10^{-9} \mathrm{erg} \mathrm{cm}^{-2} \mathrm{~s}^{-1}$ the source experienced a significant change in its spectral shape. The X-ray continuum of the brighter observations, i.e., when the $3-30 \mathrm{keV}$ flux was above $F_{\text {break }}$, showed a faster decay at high energies and required an exponential cutoff to fit the data. The energy of the cutoff increased as the flux decreased. Below $F_{\text {break }}$ this component was not needed and the X-ray continuum was well represented by a single absorbed power law extending to energies up to $100 \mathrm{keV}$ (Fig. 6). Likewise, the hydrogen column density, $N_{\mathrm{H}}$ fell rapidly as the flux decreased below $F_{\text {break }}$, while it remained at a constant value of $\sim 6.5 \times 10^{22} \mathrm{~cm}^{-2}$ above $F_{\text {break }}$. The photon index decreased as the flux increased, that is, when the source was bright, the X-ray emission was harder. At low flux, roughly coincident with $F_{\text {break }}$ the photon index flattened at a value of 1.7 (Fig. 7). Near the peak of the outburst the photon index was $\sim 1.1$.

The presence of the iron emission line or the absorption edge was not always statistically significant. When the line parameters could not be well constrained, the line and/or edge energy were fixed at their average values prior to the fit. The mean value of the central and absorption edge energies were calculated using observations in which the intensity of the line (normalization) was more than $2 \sigma$ significant and resulted in $E_{\text {line }}=6.5 \pm 0.1 \mathrm{keV}$ and $E_{\text {edge }}=9.3 \pm 0.4 \mathrm{keV}$, where the errors represent the standard deviation of all the measurements. The three bottom panels of Fig. 7 show the dependence of the iron line energy and intensity and the edge energy with the 3-30 keV X-ray continuum flux. There is a tight correlation between the X-ray continuum and the intensity of the iron line, indicating that as the illumination of the cool matter responsible for the line emission increases, so does the strength of the line.

The characteristic frequency of the broad-band noise increased as the flux increased, but saturated at $v \approx 1.5 \mathrm{~Hz}$ above $F_{\text {break }}$. The large error bars at low flux weakens the statistical significance of this trend. An almost identical trend was followed by the fractional amplitude of variability. Above $\sim 2.6 \times 10^{-9} \mathrm{erg} \mathrm{cm}^{-2} \mathrm{~s}^{-1}$ there is excess power at high frequencies, and an extra noise component with characteristic frequency at $\sim 6-7 \mathrm{~Hz}$ and $\mathrm{rms} 10-15 \%$ was needed to obtain acceptable fits (upper right panel in Fig. 6). Some of the brightest observations contain a weak QPO with centroid frequency at $\sim 1 \mathrm{~Hz}$ and $\mathrm{rms} \sim 3 \%$. Although the inclussion of this component improves the fit, an F-test shows that the probability that this improvement occurs by chance is $\sim 1-3 \%$.

\subsection{Large-scale flaring emission (MJD 53912-54799)}

At the end of the outburst decay and after a series of low-amplitude flares, Swift J1626.6-5156 began to display quasiperiodic increases in the X-ray intensity (interval $\mathrm{B}$ in Fig. 4). The maxima and minima of these flares remained roughly at the same flux level, with $F_{\max }=(28 \pm 5) \times$ $10^{-11} \mathrm{erg} \mathrm{cm}^{-2} \mathrm{~s}^{-1}$ and $F_{\min }=(5 \pm 2) \times 10^{-11} \mathrm{erg} \mathrm{cm}^{-2} \mathrm{~s}^{-1}$. The only exception was the first flare, which started and reached $\sim 2.5$ times lower and higher flux than the average minimum and maximum flux, respectively. The timescale of the X-ray modulation varied between 45 and 95 days, although most of the time separation between flares gathered around $P_{\text {orb }} / 2$ and $P_{\text {orb }} / 3$, being $P_{\text {orb }}=133$ days (see Baykal et al. 2010, for an analysis of this behaviour).

Owing to the low count rate, the energy spectra above $\sim 10 \mathrm{keV}$ and the power spectra above $\sim 1 \mathrm{~Hz}$ are too noisy for a meaningful analysis. The cutoff energy and especially the iron emission line parameters cannot be constrained in the individual spectra, which represent data obtained by integrating over $0.5-$ $1 \mathrm{ks}$, typically. However, by joining observations with roughly 

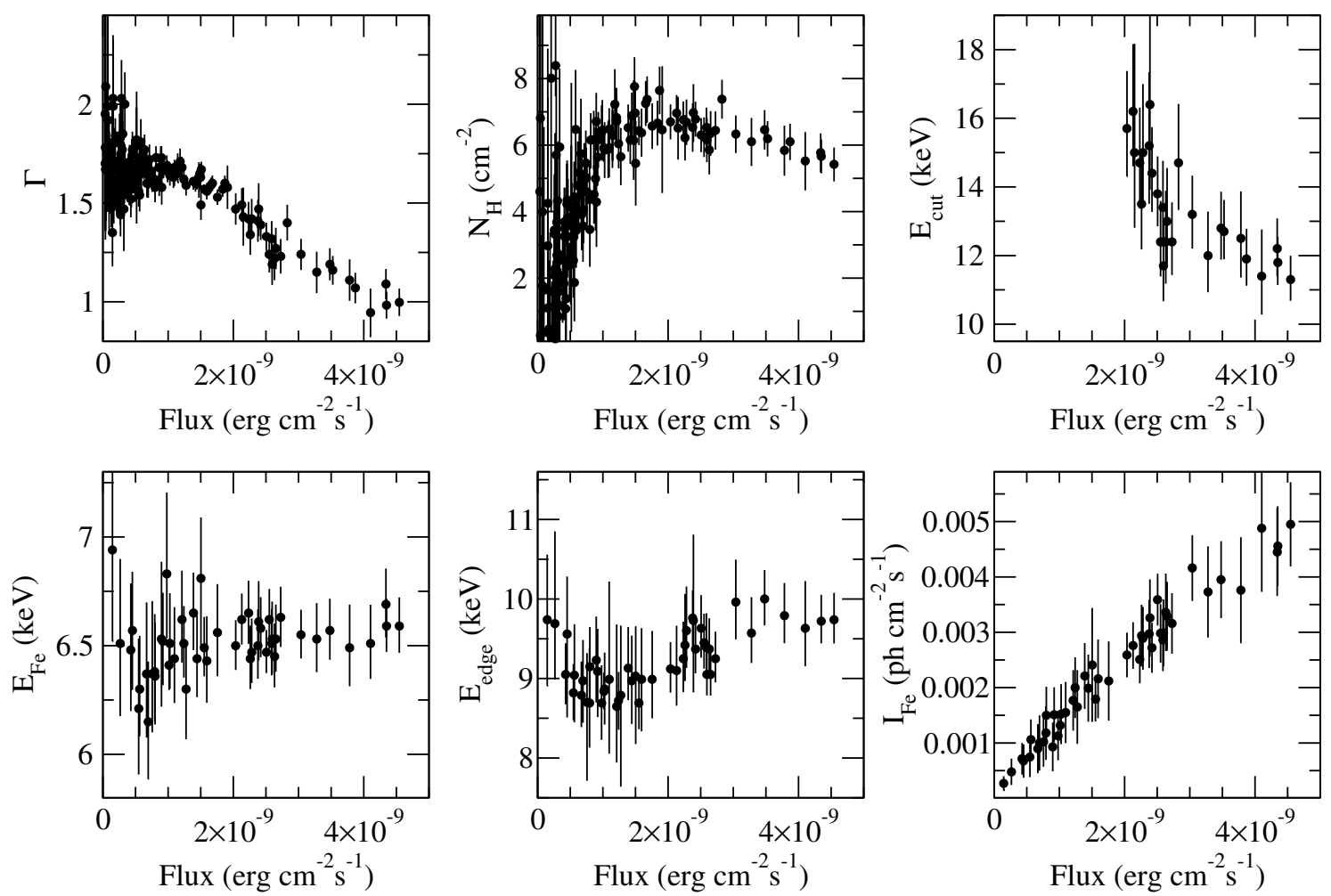

Fig. 7. Evolution of the spectral parameters over the decay of the outburst. Power-law index, hydrogen column density, cutoff energy (top panels), central energy, edge energy, and intensity of the iron line (bottom panels) as a function of X-ray flux in the 3-30 keV energy range.

Table 4. Average best-fit spectral parameters during the large-amplitude flares and the quiescent state that followed.

\begin{tabular}{|c|c|c|c|c|c|}
\hline \multirow[b]{2}{*}{ Parameter } & \multicolumn{4}{|c|}{ Flaring state } & \multirow{2}{*}{ Quiescent state } \\
\hline & Minimum & Rise & Maximum & Decay & \\
\hline Photon index $(\Gamma)$ & $1.9 \pm 0.1$ & $1.5 \pm 0.1$ & $1.16 \pm 0.09$ & $1.6 \pm 0.1$ & $2.10 \pm 0.08$ \\
\hline Cutoff energy $^{*}\left(E_{\text {cut }}\right)$ & $30^{f}$ & $20_{-3}^{+5}$ & $11 \pm 1$ & $21_{-5}^{+8}$ & $\begin{array}{c}-100.00 \\
-\end{array}$ \\
\hline Normalization $^{* *}$ & $0.017 \pm 0.001$ & $0.031 \pm 0.003$ & $0.034 \pm 0.003$ & $0.032 \pm 0.004$ & $0.007 \pm 0.001$ \\
\hline Line energy* $\left(E_{\mathrm{Fe}}\right)$ & $6.9 \pm 0.1$ & $6.7 \pm 0.1$ & $6.6 \pm 0.1$ & $6.7 \pm 0.1$ & $6.6 \pm 0.1$ \\
\hline Line width ${ }^{*}\left(\sigma_{\mathrm{Fe}}\right)$ & $0.4^{f}$ & $0.3^{f}$ & $0.2^{f}$ & $0.3^{f}$ & $0.1^{f}$ \\
\hline Edge energy* $\left(E_{\text {edge }}\right)$ & $9^{f}$ & $8.5 \pm 0.2$ & $8.3 \pm 0.1$ & $8.5 \pm 0.3$ & - \\
\hline Edge optical depth $\left(\tau_{\text {edge }}\right)$ & $0.07_{-0.07}^{+0.10}$ & $0.17 \pm 0.05$ & $0.26 \pm 0.03$ & $0.17 \pm 0.06$ & - \\
\hline$\chi^{2} /$ dof & $1.9 / 48$ & $1.6 / 46$ & $1.1 / 46$ & $1.2 / 45$ & $1.0 / 36$ \\
\hline
\end{tabular}

Notes. ${ }^{*} \mathrm{keV} ;{ }^{* *}$ ph $\mathrm{keV}^{-1} \mathrm{~cm}^{-2} \mathrm{~s}^{-1}$ at $1 \mathrm{keV} ;{ }^{f}$ fixed

the same flux, those components become significant. Therefore we obtained an averaged peaked, trough, intermediate-rise and intermediate-decay flux energy and power spectrum to increase the signal-to-noise ratio. The total on-source time for these average spectra was $17.6,19.1,15.4$ and $11.5 \mathrm{ks}$, respectively. Table 4 gives the results of the spectral fits. The spectrum at the peaks is harder than that of the troughs. The cutoff energy follows the same trend with X-ray flux as that seen during the outburst decay, namely, it increases as the flux decreases. The characteristic frequency of the broad-band noise component also agrees with the values that the source showed during the decay of the outburst for the same flux level. At the peak of the flares $v_{\max }=0.40 \pm 0.09 \mathrm{~Hz}$, while at intermediate flux $v_{\text {rise }} \approx v_{\text {decay }}=0.12 \pm 0.05 \mathrm{~Hz}$. The fractional amplitude of variability is $\sim 25 \%$ in all cases. The timing parameters at the minimum flux of the flares cannot be constrained because of the low signal-to-noise ratio of the power spectrum.

\subsection{Quiescence (MJD 54799-55544)}

After the series of flares, the source entered a quiescent state, characterised by little variability. The X-ray flux decreased to an average level of $(1.7 \pm 0.4) \times 10^{-11} \mathrm{erg} \mathrm{cm}^{-2} \mathrm{~s}^{-1}$. Again, the individual spectra are too noisy to be able to constrain the spectral parameters. Neither an iron emission line nor the exponential cutoff are statistically required in the individual spectra. However, when an average energy spectrum is obtained by combining all observations in the interval MJD 54799-55544, a good fit cannot be obtained unless a Gaussian component at $6.5 \mathrm{keV}$ is included, although no edge is required. The inclusion of that component reduced the $\chi^{2}$ statistic from 94 for 38 degrees of freedom to 35 for 36 degrees of freedom. The total on-source time of this spectrum was $46.4 \mathrm{ks}$, corresponding to 61 observations. The best-fit spectral parameters are given in Table 4 . The energy range was limited to $3-20 \mathrm{keV}$. The spectrum during the quiescent state is softer than during the outburst decay 

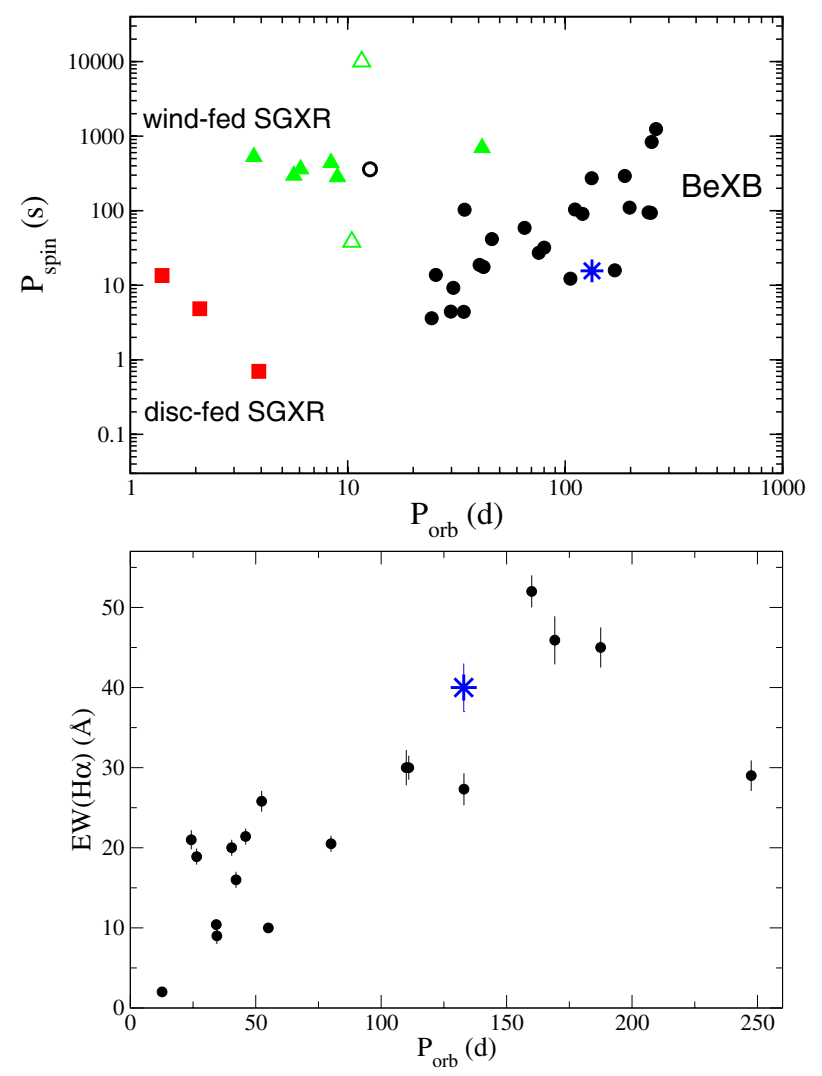

Fig. 8. $P_{\text {orb }}-P_{\text {spin }}$ and $P_{\text {orb }}-E W(H \alpha)$ diagrams. The star symbol marks the position of Swift J1626.6-5156. Open symbols in the top panel correspond to the peculiar SGXBs $2 \mathrm{~S} 0114+65$ and OAO 1657-41 and the BeXB SAX J2103.4+4545.

and during the flaring state, but follows the general trend also observed in the other two states that the lower the flux, the larger the photon index, i.e., the softer the spectrum.

\section{Discussion}

The available data in various wavelength bands provide strong evidence that the system is a Be/X-ray binary. In the X-ray band, the detection of 15.4-s pulsations and the shape of the spectral continuum (a power law with exponential cutoff) are characteristic of accreting X-ray pulsars. In the optical band, the strong $\mathrm{H} \alpha$ emission line and the presence of He I and He II lines indicate an OB-type star as the optical counterpart. In the NIR, both the $H$ - and the $K$-band observed features (HeI end $\mathrm{HI}$ recombination series in emission) are also typical of early Be stars. This allows us to classify the counterpart to Swift J1626.6-5156 as a B0Ve star, and the system as a Be/X-ray binary.

Additional evidence in favour of a Be/X-ray binary classification comes from the $P_{\text {orb }}-P_{\text {spin }}$ diagram (Corbet 1986) and the $P_{\text {orb }}-E W(H \alpha)$ diagram (Reig et al. 1997; Reig 2011). Figure 8 shows these two diagrams. As can be seen, Swift J1626.6-5156 (big star symbol) agrees with the $P_{\text {orb }}-E W(H \alpha)$ correlation and falls in the Be/X-ray binaries region of the $P_{\text {orb }}-P_{\text {spin }}$ diagram.

The optical spectra that cover the region of the $\mathrm{H} \alpha$ line show comparable values of the $\mathrm{H} \alpha$ equivalent width even though they correspond to very different X-ray states and were taken years apart. The February 2006 observation corresponds to the middle of the X-ray outburst decay, the September 2007 observations correspond to the middle of the flaring episode and the June 2009 observation to the beginning of the quiescent X-ray state. In Be stars, the strength and shape of the $\mathrm{H} \alpha$ line provides information about the physical conditions in the circumstellar disc. Generally, after a major X-ray outburst the $\mathrm{H} \alpha$ equivalent width decreases substantially, which indicates a weakening of the disc (Coe et al. 1994). The line shape may even turn from an emission profile to an absorption profile, suggesting that the disc is lost (Reig et al. 2007). In contrast, the disc in Swift J1626.65156 does not seem to be affected by the X-ray outburst, because the $\mathrm{H} \alpha$ equivalent width did not vary much immediately after the $\mathrm{X}$-ray outburst or a few years later.

The behaviour of Swift J1626.6-5156 resembles that of the Be/X-ray binary KS 1947+300. Like Swift J1626.6-5156, KS $1947+300$ went through a series of periodic increases of the X-ray intensity (type I outburst) after a major (type II) outburst (see Fig. 11 in Reig 2011) without the $\mathrm{H} \alpha$ line beeing affected. Another characteristic in common with KS 1947+300 is the near-circular orbit. The eccentricity in these two systems $(0.03$ and 0.08 , respectively) are among the smallest in Be/X-ray binaries. An alternative scenario would be that of 1A 1118-61, which exhibited a huge equivalent width ( $-90 \AA$ ) before the X-ray outburst (Coe et al. 1994). After the outburst, the $\mathrm{H} \alpha$ equivalent width decreased substantially but remained at a relatively high level around $-60 \AA$ for the following years (Villada et al. 1999).

A discrete component at $\sim 6.5 \mathrm{keV}$ is always present, albeit weak, in the X-ray energy spectra of Swift J1626.6-5156 in all states. This component is interpreted as reprocessing of the hard $\mathrm{X}$-ray continuum in relatively cool matter. Near-neutral iron generates a line centered at $6.4 \mathrm{keV}$, while this energy increases as the ionisation stage increases. However, the energy separation is so small that even ionised iron up to Fe XVIII can be thought as part of the $6.4 \mathrm{keV}$ blend (Liedahl 2005). The line energy during the outburst decay is consistent with this neutral iron blend. The line energy during the flaring state seems to be higher. However, given the weakness of the line, we do not claim a higher ionisation degree in this state. What it is important to notice here is that the fluorescent iron line provides strong evidence for the presence of material in the vicinity of the X-ray source. It has been detected in virtually all high-mass X-ray binaries with supergiant companions. In these systems the Fe line fluorescence is produced in the stellar wind of the massive star (Torrejón et al. 2010). The possible site for reprocessed emission in a Be/X-ray binary are an accretion disc or the circumstellar (decretion) disc. The former surrounds the neutron star, while the latter surrounds the Be star's equator. The detection of this component in the energy spectrum of the quiescent state would favour the equatorial disc as the site for the reprocessing of hard-energy photons, because we would expect that the accretion disc, if present at all, would have disappeared. The alternative scenario that the Fe iron line emission is produced in the thin thermal hot plasma that is presumably located along the Galactic plane (Yamauchi et al. 2009 ) is ruled out by the observed correlation between the line intensity and the X-ray continuum flux.

We estimate the distance to be $\sim 10 \mathrm{kpc}$ with an error of the order of $30 \%$. This distance and the Galactic coordinates of the source $(l=332.8, b=-2.0)$ place the system in the Norma Arm of the Galaxy. The sources of error in the determination of the distance can be attributed to i) uncertainties in the calibrations that give intrinsic colours and absolute magnitudes as a function of spectral types; ii) the presence of a large circumstellar disc which contaminates the optical and IR colours making the observed colours appear redder that their intrinsic values, and to a lesser extent; iii) the uncertainty in the spectral type.

Note that in Be stars the total reddening not only includes the contribution of the interstellar extinction but also of the 
reddening caused by the circumstellar material. The contribution of the circumstellar disc to the photometric colours increases with wavelength from $\sim 0.1 \mathrm{mag}$ in $(B-V)$ to $\sim 0.8 \mathrm{mag}$ in $(J-M)$ for well-developed discs (Dachs et al. 1988). Moreover, it has been shown that the circumstellar reddening correlates with the equivalent width of the $\mathrm{H} \alpha$ line (Dachs et al. 1988; Fabregat \& Torrejón 1998; Howells et al. 2001). The broader the equivalent width, the larger the contribution of the circumstellar emission to the total reddening. Because Swift J1626.6-5156 shows strong $\mathrm{H} \alpha$ emission with an equivalent width of $-45 \AA$, the disc emission can make an important contribution to the colour excess. Although we try to account for this extra extinction using the equations by Howells et al. (2001), it is worth noticing that the photometric infrared magnitudes and the optical and infrared spectra were not contemporaneous, which introduces extra uncertainty in the distance estimation given the high-amplitude variability that characterises the optical and infrared emission of Be stars. Our distance estimate is consistent with the value of $\sim 15 \mathrm{kpc}$ reported by Icdem et al. (2011). These authors estimated the distance from the correlation between spin-up rate and X-ray flux. Although Icdem et al. (2011) do not give the error of their measurement, the application of this method in other sources has shown that the uncertainty is of the order of $\sim 20 \%$.

\section{Conclusion}

We have solved the nature of the X-ray pulsar Swift J1626.65156. The new optical and infrared data provide strong evidence that the system is a Be/X-ray binary. The location of Swift J1626.6-5156 in the $P_{\text {orb }}-P_{\text {spin }}$ and $P_{\text {orb }}-E W(H \alpha)$ diagrams, the X-ray pulsations, the shape of the X-ray continuum, the longterm X-ray variability, the emission of the Balmer and Brackett lines in the optical/IR spectrum are typical characteristics of high-mass X-ray binaries with a Be companion. We conclude that Swift J1626.6-5156 is a Be/X-ray binary with a B0Ve optical counterpart located at a distance of $\sim 10 \mathrm{kpc}$. Optical photometric observations are needed to reduce the uncertainty in the distance estimation.

Acknowledgements. We thank the referee, Malcolm Coe, for his useful and important comments that improved the final version of this manuscript. The work of E.N. y J.F. is supported by the Spanish Ministerio de Educación y Ciencia, and FEDER, under contract AYA2010-18352. P.R. and J.F. are partially supported by the Generalitat Valenciana project of excellence PROMETEO/2009/064. PR also acknowledges partial support by the COST Action ECOST-STSM-MP0905280611-008632. R.E.M. acknowledges support by Fondecyt grant 1070705, 1110347, the Chilean Center for Astrophysics FONDAP 15010003 and from the BASAL Centro de Astrofísica y Tecnologias Afines (CATA) PFB-06/2007. This work was based on observations collected at the European Southern Observatory, Chile (Programme ID: 085.D-0297A) and on data obtained from the ESO Science Archive Facility. This research has made use of the USNOFS Image and
Catalogue Archive operated by the United States Naval Observatory, Flagstaff Station (http://wwW . nofs . navy.mil/data/fchpix/).

\section{References}

Antoniou, V., Hatzidimitriou, D., Zezas, A., \& Reig, P. 2009, ApJ, 707, 1080

Baykal, A., Gögus, E., Inam, S.Ç., \& Belloni, T. 2010, ApJ, 711, 1306

Baykal, A., Inam, S. C., \& Icdem B. 2011, Proceedings of Astrophysics of Neutron Stars 2010 - a Conference in honor of M. Ali Alpar, 2-6 August 2010, Cesme, Izmir [arXiv: 1104.4432]

Belloni, T., \& Hasinger, G. 1990, 230, 103

Belloni, T., Homan, J., Campana, S., Markwardt, C. B., \& Gehrels, N. 2006, ATel 687

Bradt, H. V., Rothschild, R. E., \& Swank, J. H. 1993, A\&AS, 97, 355

Campana, S., Belloni, T., Homan, J., et al. 2006, ATel 688

Clark, J. S., \& Steele, I. A. 2000, A\&AS, 141, 65

Coe, M. J., Roche, P., Everall, C., Fishman, G. J., \& Hagedon, K. S. 1994, A\&A, 289, 784

Corbet, R. H. D. 1986, MNRAS, 220, 1047

Dachs, J., Engels, D., \& Kiehling, R. 1988, A\&A, 194, 167

Dougherty, S. M., Waters, L. B. F. M., Burki, et al. 1994, A\&A, 290, 609

Draine, B. T. 1989, ESA SP-290, 93

Fabregat, J., \& Torrejon, J. M. 1998, A\&A, 332, 643

Güver, T., \& Öxel, F. 2009, MNRAS, 400, 2050

Howells, L., Steele, I. A., Porter, J. M., \& Etherton, J. 2001, A\&A, 369, 99

Icdem, B., Inam, S. C., \& Baykal, A. 2011, MNRAS, 415, 1523

Jaschek C., \& Gómez A. E. 1998, A\&A, 330, 619

Johnson, H. 1966 ARA\&A, 4, 193

Koornneef, J. 1983, A\&A, 128, 84

Liedahl, D. A. 2005, AIPC, 774, 99

Markwardt, C. B., \& Swank, J. H. 2005, ATel 679

Miyamoto, S., Kimura, K., Kitamoto, S., Dotani, T., \& Ebisawa, K. 1991, ApJ, 383,784

Moorwood, A., Cuby, J.-G., \& Lidman, C. 1998, The Messenger, 91, 9

Negueruela, I., \& Marco, A. 2006, ATel 739

Nowak, M. A., Vaughan, B. A., Wilms, J., Dove, J. B., \& Begelman, C. 1999, ApJ, 510, 874

Palmer, D., Barthelmy, S., Cummings, J., et al. 2005, ATel 678

Rea N., Testa V., Israel G. L., et al. 2006, ATel 713

Reig, P. 2011, Ap\&SS, 332, 1

Reig, P., Fabregat, J., \& Coe, M. J. 1997, A\&A, 322, 193

Reig, P., Negueruela, I., Fabregat, J., Chato, R., \& Coe, M. J., 2005, A\&A, 440, 1079

Reig, P., Larionov, V., Negueruela, Arkharov, A. A., \& Kudryavtseva, N. A. 2007, A\&A, 462, 1081

Reig, P., Belloni, T., Israel, G. L., et al. 2008, A\&A, 485, 797

Rieke, G. H., \& Lebosfsky, M. J. 1985, ApJ, 288, 618

Steele, I. A., \& Clark, J. S. 2001, A\&A, 371, 643S

Tarana, A., Bazzano, A., Chenevez, J., et al. 2006, ATel, 739

Torrejón, J. M., Schulz, N. S., Nowak, M. A., \& Kallman, T. R. 2010, ApJ, 715, 947

Villada, M., Rossi, C., Polcaro, V. F., \& Giovannelli, F. 1999, A\&A, 344, 277

Walborn, N. R., \& Fitzpatrick, E. L., 1990, PASP, 102, 379

Wegner, W. 1994, MNRAS, 270, 229

Wegner, W. 2006, MNRAS, 371, 185

Wilson, C. A., Finger, M. H., Coe, M. J., Laycock, S., \& Fabregat, J., ApJ, 570, 287

Yamauchi, S., Ebisawa, K., Tanaka, Y., et al. 2009, PASJ, 61, S225 